\title{
Discriminative Hypomania Checklist-32 Factors in Unipolar and Bipolar Major Depressive Patients
}

\author{
Giulio Perugia, $^{a}$ Michele Fornaro ${ }^{c}$ Icro Maremmani $^{a}$ b Pier Luigi Canonico ${ }^{d}$ \\ Paolo Carbonatto ${ }^{e}$ Claudio Mencacci $^{f}$ Giovanni Muscettolag Luca Pani ${ }^{h}$ \\ Riccardo Torta ${ }^{i}$ Claudio Vampini ${ }^{j}$ Fabio Parazzini ${ }^{k}$ Arina Dumitriul \\ Jules Angst ${ }^{m}$ on behalf of Come To Me Study Group \\ a Department of Psychiatry, University of Pisa, and 'Institute of Behavioral Sciences 'G. De Lisio', Pisa, 'Section of \\ Psychiatry, Department of Neurosciences, University of Genova, Genoa, 'University of Novara, Novara, ${ }^{\mathrm{e}}$ Italian \\ Society of General Practitioners (SIMG), Turin, ${ }^{f}$ Fatebenefratelli Hospital, Milan, 9University of Napoli, Naples, \\ hInstitute of Neurogenetics and Neuropharmacology, CNR, Cagliari, 'Molinette Hospital, Turin, 'jMaggiore Hospital,

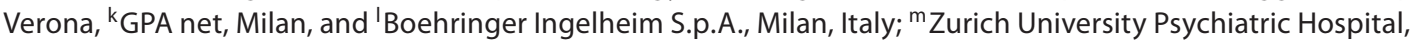 \\ Zurich, Switzerland
}

\section{Key Words}

Hypomania Checklist-32 $\cdot$ Bipolar disorder $\cdot$ Depression

\begin{abstract}
Background: Although manic or hypomanic episodes define bipolar disorder (BD), most patients show a predominance of depressive symptomatology, often associated with delayed or disregarded BD diagnosis. The Hypomania Checklist-32 (HCL-32) has therefore been developed and tested internationally to facilitate BD recognition. Sampling and Methods: Five hundred seventy-one (563 eligible) patients diagnosed with a major depressive episode according to DSM-IV criteria were consecutively enrolled in a cross-sectional, multicenter, observational study (Come To Me). Lifetime manic or hypomanic features were assessed by the HCL-32, and severity of depressive and anxious symptomatology was assessed using the Zung's self-report questionnaires for depression and anxiety. Results: Among the patients diagnosed with BD ( $n=119)$, either type I or type II, the occurrence of (hypo)manic symptoms was significantly higher compared to major depressive disorder (MDD) symptoms according to $\mathrm{HCL}-32$ total and subscale scores obtained
\end{abstract}

(C) 2012 S. Karger AG, Basel

0254-4962/12/0456-0390\$38.00/0

Fax +41613061234

E-Mail karger@karger.ch

www.karger.com
Accessible online at: www.karger.com/psp using a score of 14 , which ensured an optimal discrimination between $B D$ and MDD with a sensitivity of 0.85 and a specificity of 0.78 . Conclusions: Although some false positives might occur, the HCL-32 was confirmed to be a useful instrument in the detection of past hypomania in MDD patients, finally contributing to proper therapeutic choices.

Copyright $\odot 2012$ S. Karger AG, Basel

\section{Introduction}

Bipolar disorder (BD) is a complex chronic condition characterized by recurring depressive, (hypo)manic and/ or mixed episodes [1]. Although the manic or hypomanic episodes define the disorder, the majority of patients observed in the clinical setting is characterized by a predominance of depressive symptoms over manic ones, accounting for much of the morbidity and mortality associated with $\mathrm{BD}[2,3]$. For this reason, in many cases, diagnosis relies on careful clinical assessment of past (hypo) manic episodes [4], while 'correct' diagnosis and treatment for BD often lags many years [5]. In fact, despite careful history taking, it may be difficult to get a conclu- 
sive diagnosis in the course of an acute episode. For example, a depressed patient may report 'always' being depressed and neglect to recall periods of mood elation or irritability, which are consistent with hypomania $[6,7]$. In such a scenario, differential diagnosis is crucial. Among axis I disorders, major depressive disorder (MDD) is the most critical differential diagnosis. A correct BD diagnosis should not leave aside a careful questioning about past (hypo)manic symptoms [8]. In fact, cross-sectional evaluation of depressive symptoms cannot be reliably used for the differential diagnosis. Atypical depressive symptoms such as hypersomnia, carbohydrate craving, 'reverse' neurovegetative signs and interpersonal sensitivity have been frequently reported in bipolar II depression $[9,10]$, but these features are common in MDD as well [11]. Some authors suggest that irritability during depressive episodes should be a marker of bipolarity [12], but this feature is insufficient to make BD diagnosis. Similarly, psychotic symptoms may be more common across $\mathrm{BD}$ rather than MDD, but they are not highly specific [13].

Recently, an upsurge of interest in understanding the burden of bipolar depression [14] has favored the development of a number of tools aiming to facilitate diagnosis. Among them, the Mood Disorder Questionnaire (MDQ) has shown a poor sensibility compared to the Structured Clinical Interview for Diagnosis (SCID) [15], and the Bipolar Spectrum Diagnostic Scale (BSDS), also using a self-report screening too, has shown different performances depending on the screened population [16] with cross-sectional evaluation of depressive symptoms being substantially of little use in detecting BD [17]. The MDQ has been widely studied $[15,18]$ and it has been shown to be sensitive to identify BD type I (BD-I) but probably less adequate for BD type II (BD-II) [19-21]. The instrument was first tested in a tertiary care clinical setting showing a sensitivity of $73 \%$ and a specificity of $90 \%$ (two-thirds of the sample were BD-I); in a general population sample, however, the sensitivity was $28 \%$ and specificity $97 \%$ [22].

The Hypomania Checklist-32 (HCL-32) is a self-report screening tool developed to identify bipolarity in epidemiological and clinical populations aiming to help the clinician to diagnose BD-II and other bipolar spectrum disorders [23] in patients with DSM-IV-defined MDD. The original aim was to develop a short multilingual version of a robust self-assessment screening tool for BD that could be used across countries and continents. Indeed, in recent studies [24] HCL-32 has shown good discriminating power between the unipolar and bipolar samples. A cutoff of 14 offered the best trade-off between sensitivity ('true bipolars') and specificity ('true non-bipolars') with the total scale showing a sensitivity of $80 \%$ and a specificity of $51 \%$ for both types of $\mathrm{BD}[24,25]$. The sensitivity is slightly higher and specificity slightly lower than reported for the MDQ in a clinical setting. However, it may be an advantage for a screening tool to have higher sensitivity than specificity. Anyway, the above-mentioned study showed HCL-32 might help distinguish between MDD and $\mathrm{BD}$, but it seems to be unable to distinguish BD-I and BD-II disorders [24]. Furthermore, the scale had a positive predictive value of $73 \%$ and a negative predictive value of $63 \%$, suggesting that the discriminatory power of the scale would be maintained at an acceptable level when the scale is employed in community settings, where overall rates of $\mathrm{BD}$ would be lower than that expected in clinical settings. Interestingly, according to Angst et al. [24], current mental state seems to have no impact on the HCL-32 self-assessment of hypomanic symptoms, thus making the scale suitable for screening purposes even for patients presenting current symptomatic depressive states [26].

Analyses of Italian, Swedish, Spanish and Chinese clinical samples independently gave similar two-factor structures of HCL-32, an 'active/elated' factor and a 'risktaking/irritable' one. The active/elated factor included mainly hyperactivity (which is already proposed as a core feature in BD-II patients [27]), mood elation and improved thinking. The risk-taking/irritable factor included symptoms of risk-taking behavior, anger/irritability and flight of ideas. This factor structure is comparable to the one described by Hantouche et al. [28] on the basis of the original shorter version of the Hypomania Checklist (HCL-20) used in the French multicenter EPIDEP study and in Danish samples [29]. It is also in line with the factor analysis of hypomania based on the MDQ [26]. Analogously, a similar two-factorial structure has been observed in a normal population [24]. On the other hand, the factorial validation of the recently proposed Polish version of HCL-32 submitted to 150 major depressive patients yielded three factors: factor 1 (ten items associated with elevated mood and increased activity) explained more than half of total variance, factor 2 (two items related with sexual activity) explained $11 \%$ of variance and factor 3 (three items linked with irritability) explained only $3 \%[30]$.

The aim of the present study is to explore the factorial structure of HCL-32 in a large national sample of patients enrolled in a study exploring the presence of somatic symptoms during major depression [31] and to analyze the discriminative power of different factors in distinguishing non-bipolar from bipolar patients. Another goal 
of the study is to define some of the characteristics identified by the HCL-32, but not recognized by the patients (depending on the patient's ability to recall hypomanic episodes and on the patient's awareness that they represent part of the affective illness) or alternatively by the clinicians (depending on the clinician's attitude in interviewing patients and in the interpretation of the hypomanic manifestations).

\section{Method}

The present study is based on a post-hoc partial analysis of the database collected for a previous study including 571 consecutive patients with a diagnosis of an major depressive episode (MDE), according to DSM-IV, who were enrolled in a large cross-sectional, multicenter, observational study (Come To Me) [31]. The study involved 30 psychiatric outpatient facilities, distributed throughout Italy, assessing the impact of medically unexplained somatic symptoms in course of an MDE and its relationship to the severity of depressive symptomatology, the risk for misdiagnosis and associated improper treatment and health resource overutilization. Both Kaiser-Meyer-Olkin measure of sampling adequacy tests and Bartlett's test of sphericity indicated that the two-factor model was appropriate. The PCA method was chosen since it is sensitive to the relative scaling of the original variables. Oblique rotation was applied to simplify the interpretation of factors.

According to the observational nature of the protocol, routine medical procedures were not modified. The ethics committee of each center approved the study protocol in accordance with the Italian Ministerial Bulletin issued on September 2, 2002 regarding observational studies. Finally, the study was sponsored by Boehringer Ingelheim Italy and all patients gave their informed consent concerning handling and use of the data collected during the course of the study.

\section{Study Population}

Subjects referred to the selected centers between December 2006 and July 2007 were screened for inclusion in the study. Patients were recruited consecutively according to the following inclusion criteria: (1) both genders, aged 18-75 years; (2) diagnosis of an MDE according to DSM-IV (MDD, recurrent major depression, depressive episode in course of BD-I and BD-II, depression NOS); and (3) ability to complete the self- and heteroadministered questionnaires. The exclusion criteria were: (1) comorbidity with schizophrenia and other psychotic disturbances, and (2) current relevant physical illnesses.

The study included 571 depressed outpatients, but 8 (1.4\%) of them provided incomplete information on demographic and clinical variables. The study population therefore consisted of 563 eligible subjects with a mean number of patients of 18.7 (range $5-56)$ for each center; 376 subjects $(66.8 \%)$ were females and 187 (33.2\%) males. Regarding age, 251 subjects (44.5\%) were 18 - to 50 -years-old and 313 (55.5\%) were over 50 years of age; mean age was 51 years $(\mathrm{SD}=13)$. The level of education was higher than 11 years for $245(43.5 \%)$ patients and most of them $(n=331,58.8 \%)$ were married.
Diagnostic Procedure and Symptom Assessment

Individuals were assessed by psychiatrists with extensive clinical experience in the diagnosis and treatment of mood disorders. Diagnosis of MDE (MDD, recurrent major depression, depressive episode types BD-I and BD-II; depression NOS) was made according to DSM-IV criteria.

An anonymous data sheet was used to collect the following information: demographic status; lifestyle; surgical, pathologic and pharmacological case history; presence and characterization of somatic symptoms; related delay in major depression diagnosis and treatment; and consumption of health resources in the 6 months preceding enrollment. All the information were gathered directly by the patient with the help of significant others and medical records.

The HCL-32 [24] was administered for the evaluation of lifetime manic or hypomanic features and severity of depression; anxious symptomatology was self-evaluated by means of the Zung's questionnaires for depression (Zung-D) and anxiety (Zung-A) [32, 33].

\section{Statistics}

Tetrachoric correlation matrix of HCL-32 items was evaluated. Exploratory factor analysis was then performed in order to explore the factor structure of the correlation matrix. Oblique rotation was applied to simplify the interpretation of factors. Eigenvalues $>1$ were initially retained, but clinical considerations also decided the final number of factors. Subscale scores for each factor were obtained by summing all items that loaded higher than 0.4 on the corresponding factor.

Reliability of the Italian version of HCL-32 and its subscales was examined using Cronbach's (1951) coefficient alpha. Associations between scores on the Zung-D, Zung-A and HCL-32 total and factor scores were evaluated by Pearson's correlations.

The accuracy of the HCL-32 was calculated in terms of sensitivity and specificity yielded by a receiver operating characteristic analysis for each cutoff point. To study the sensitivity, specificity and efficacy of the HCL-32, the proportion of subjects correctly diagnosed with BD and MDD was calculated.

A discriminant analysis was then used to describe the percentage of MDE subjects correctly classified as bipolar and non-bipolar based on the result obtained on the HCL-32 factor scores. The term 'classification' indicates the process of identifying the likely group membership of a case when the only information known is the case values on the discriminating variables. This facet of discriminant analysis was used in testing the adequacy of the derived discriminant functions. By classifying the cases used to derive the functions in the first place and comparing predicted group membership with actual group membership, one can empirically measure success in discrimination by observing the proportion of correct classifications. From the classification table, one can also observe whether the errors tend to fall into certain groups.

\section{Results}

The factor analysis initially yielded four factors with eigenvalues $>1$, explaining $58.8 \%$ of the variance. However, according to the scree test, a 2 -factor solution was 
Table 1. Principal component analysis (varimax rotation) of the HCL-32

\begin{tabular}{|c|c|c|c|}
\hline & & Factor 1 & Factor 2 \\
\hline 1 & I need less sleep & 0.51 & 0.29 \\
\hline 2 & I feel more energetic and more active & 0.82 & -0.06 \\
\hline 3 & I am more self-confident & 0.78 & -0.05 \\
\hline 4 & I enjoy my work more & 0.72 & -0.03 \\
\hline 5 & I am more sociable (make more phone calls, go out more) & 0.79 & 0.07 \\
\hline 6 & I want to travel and/or travel more & 0.58 & 0.29 \\
\hline 7 & I tend to drive faster or take more risks & 0.23 & 0.48 \\
\hline 8 & I spend more/too much money & 0.36 & 0.48 \\
\hline 9 & I take more risks in my daily life & 0.34 & 0.51 \\
\hline 10 & I am physically more active (sport, etc.) & 0.71 & 0.05 \\
\hline 11 & I plan more activities or projects & 0.76 & 0.11 \\
\hline 12 & I have more ideas. I am more creative & 0.79 & 0.12 \\
\hline 13 & I am less shy or inhibited & 0.61 & 0.25 \\
\hline 14 & I am more 'colorful' and I wear more extravagant clothes/make up & 0.37 & 0.33 \\
\hline 15 & I want to meet or actually do meet more people & 0.74 & 0.09 \\
\hline 16 & I have more interest in sex & 0.60 & 0.24 \\
\hline 17 & I am more flirtatious and/or more sexually active & 0.52 & 0.31 \\
\hline 18 & I talk more & 0.68 & 0.22 \\
\hline 19 & I think faster & 0.67 & 0.34 \\
\hline 20 & I make more jokes & 0.63 & 0.27 \\
\hline 21 & I am more easily distracted & -0.10 & 0.57 \\
\hline 22 & I engage in lots of new things & 0.58 & 0.20 \\
\hline 23 & My thoughts jump from topic to topic & 0.30 & 0.56 \\
\hline 24 & I do things more quickly and/or more easily & 0.78 & 0.12 \\
\hline 25 & I am more impatient and/or get irritable & 0.03 & 0.68 \\
\hline 26 & I can be exhausting or irritating for others & 0.00 & 0.72 \\
\hline 27 & I get into more quarrels & 0.08 & 0.61 \\
\hline & My mood is higher, more optimistic & 0.75 & -0.04 \\
\hline & I drink more coffee & 0.19 & 0.49 \\
\hline & I smoke more cigarettes & 0.12 & 0.48 \\
\hline & I drink more alcohol & 0.10 & 0.41 \\
\hline & I take more drugs (sedatives, anxiolytics, stimulants) & -0.23 & 0.42 \\
\hline \multicolumn{2}{|r|}{ Eigenvalue } & 10.77 & 3.30 \\
\hline \multicolumn{2}{|c|}{$\%$ variance } & 33.7 & 10.3 \\
\hline
\end{tabular}

The components of each factor, including the items with components greater than 0.40 , indicate that factor 1 would comprise 19 items (items $1,2,3,4,5,6,10,11,12,13,15,16,17,18,19,20,22,24$ and 28) and factor 2 would comprise 12 items (items 7, 8, 9, 23, 25, 26, 27, 29, 30, 31 and 32).

preferred. The two-factor solution explains a smaller proportion of variance than the four-factor solution, the one adopted by the Farsi version of the HCL-32 [34]; nonetheless it was preferred because it was simpler, more consistent with clinical experience and had previous reports. Obviously, this procedure was prone to 'a researcher-controlled bias'. The eigenvalues of the two factors, labeled 'hyperactive/elated' and 'irritable/distractible/impulsive' hypomania, were 10.8 and 3.3, respectively, and together accounted for $44.0 \%$ of the total variance. The components of each factor, including the items with components greater than 0.40 , indicate that factor 1 would comprise 19 items (items $1,2,3,4,5,6,10,11,12,13,15,16,17,18$, $19,20,22,24$ and 28 ) and factor 2 would comprise 12 items (items 7, 8, 9, 21, 23, 25, 26, 27, 29, 30, 31 and 32; table 1).

The internal consistency was evaluated for the entire HCL-32. The coefficient of internal consistency was high, with a Cronbach's alpha of 0.94 , indicating a high level of homogeneity of the questionnaire's items. The majority of item-total scale correlations ranged from 0.40 to 0.81 . The elimination of each item did not lead to a substantial 
Table 2. Comparison between diagnostic subtypes in demographic and symptomatological features

\begin{tabular}{|c|c|c|c|c|c|}
\hline Age & $51 \pm 13$ & $52 \pm 14$ & $50 \pm 12$ & & \\
\hline Sex, female & $376(66.8 \%)$ & $306(69.9 \%)$ & $70(58.8 \%)$ & 4 & 0.030 \\
\hline Single & $232(41 \%)$ & $181(40.7 \%)$ & $51(42.8 \%)$ & 1.01 & 0.653 \\
\hline HCL-32 total score & $12.70 \pm 8.1$ & $10.81 \pm 7.5$ & $19.74 \pm 6.4$ & -12.94 & 0.000 \\
\hline Zung-D total score & $53.16 \pm 9.0$ & $53.29 \pm 9.0$ & $52.64 \pm 9.4$ & 0.67 & 0.501 \\
\hline Zung-A total score & $47.00 \pm 9.9$ & $47.77 \pm 9.9$ & $44.13 \pm 9.3$ & 3.73 & 0.000 \\
\hline
\end{tabular}

increase in the questionnaire's internal consistency. Cronbach's alpha for factor 1 and factor 2 were 0.94 and 0.79 , respectively.

There were no significant age and gender differences in HCL-32 total or factor subscale scores (all $p$ values $>0.10)$. The patients diagnosed with BD reported significantly more (hypo)manic symptoms than those diagnosed MDD, according to the total and subscale scores obtained (table 2). As expected, both factors scales resulted higher in BD than in MDD patients. The Zung-D total score was similar in bipolar and non-bipolar subjects. These latter reported higher Zung-A total scores in comparison with $\mathrm{BD}$ patients.

We analyzed the scale's discriminative capacity for $\mathrm{BD}$ by means of a receiver operating characteristic curve (fig. 1). The area under the curve is 0.82 , indicating that the instrument's capacity is good and near to 1 . A score of 14 provides the best balance between sensitivity and specificity and discriminates between $\mathrm{BD}$ and $\mathrm{MDD}$ with a sensitivity of 0.81 (95\% CI: $0.65,0.90)$ and a specificity of 0.64 (95\% CI: 0.69, 0.82). A HCL-32 score of 14 or more was reported by 175 (39.4\%) of our MDD and 98 $(82.4 \%)$ of our BD cases defined according to DSM-IV criteria.

Concerning the discriminant analysis (table 3), the identified function significantly differentiates MDD from BD patients. HCL-32 factor 2 ('irritable/distractible/ impulsive') has the highest discriminative power, while factor 1 ('hyperactive/elated') the lowest. The automatic reclassification of the diagnostic groups shows that $75.8 \%$ of the subjects were correctly classified; $76.4 \%$ of MDD and $73.9 \%$ of $\mathrm{BD}$ subjects were reclassified in the same predicted group.
Table 3. Discriminant analysis between diagnostic subtypes according to HCL-32 extracted factors

\begin{tabular}{lllcl}
\hline & $\begin{array}{l}\text { Non-bipolar } \\
\text { MDE } \\
(\mathrm{n}=444)\end{array}$ & $\begin{array}{l}\text { Bipolar } \\
\text { MDE } \\
(\mathrm{n}=119)\end{array}$ & $\mathrm{F}$ & $\begin{array}{l}\text { Discrimi- } \\
\text { nant } \\
\text { function }\end{array}$ \\
\hline Factor 1 & $-0.15 \pm 1.0$ & $0.56 \pm 0.7$ & $52.71^{*}$ & 0.65 \\
Factor 2 & $-0.20 \pm 0.9$ & $0.75 \pm 0.9$ & $101.5^{*}$ & 0.84 \\
Group centroids & -0.28 & 1.08 & & \\
\hline
\end{tabular}

\begin{tabular}{|c|c|c|c|}
\hline \multirow[t]{2}{*}{ Actual group } & \multirow[t]{2}{*}{ Cases, $\mathrm{n}$} & \multicolumn{2}{|c|}{ Predicted group membership } \\
\hline & & $\begin{array}{l}\text { non-bipolar } \\
\text { MDE } \\
(n=370)\end{array}$ & $\begin{array}{l}\text { bipolar } \\
\text { MDE } \\
(n=193)\end{array}$ \\
\hline MDE non-bipolar & 444 & $339(76.4 \%)$ & $105(23.6 \%)$ \\
\hline MDE bipolar & 119 & $31(26.1 \%)$ & $88(73.9 \%)$ \\
\hline
\end{tabular}

Percent of grouped cases correctly classified: $75.84 \%(n=427)$. ${ }^{*} \mathrm{p}<0.001$.

${ }^{1}$ Wilks' lambda $=0.76 ; \chi^{2} 153.02 ;$ d.f. $2 ; \mathrm{p}<0.001$.

\section{Discussion}

In our sample, the internal structure of the HCL-32 shows results similar to those obtained in prior studies conducted in European [24, 25,35] and Chinese [36] clinical samples in different symptomatic phases. The factorial solution in these studies explained $26.8-44.5 \%$ of the variance. Our two-factor solution explains $44 \%$ of variance. The factor structure in this sample is also compa- 


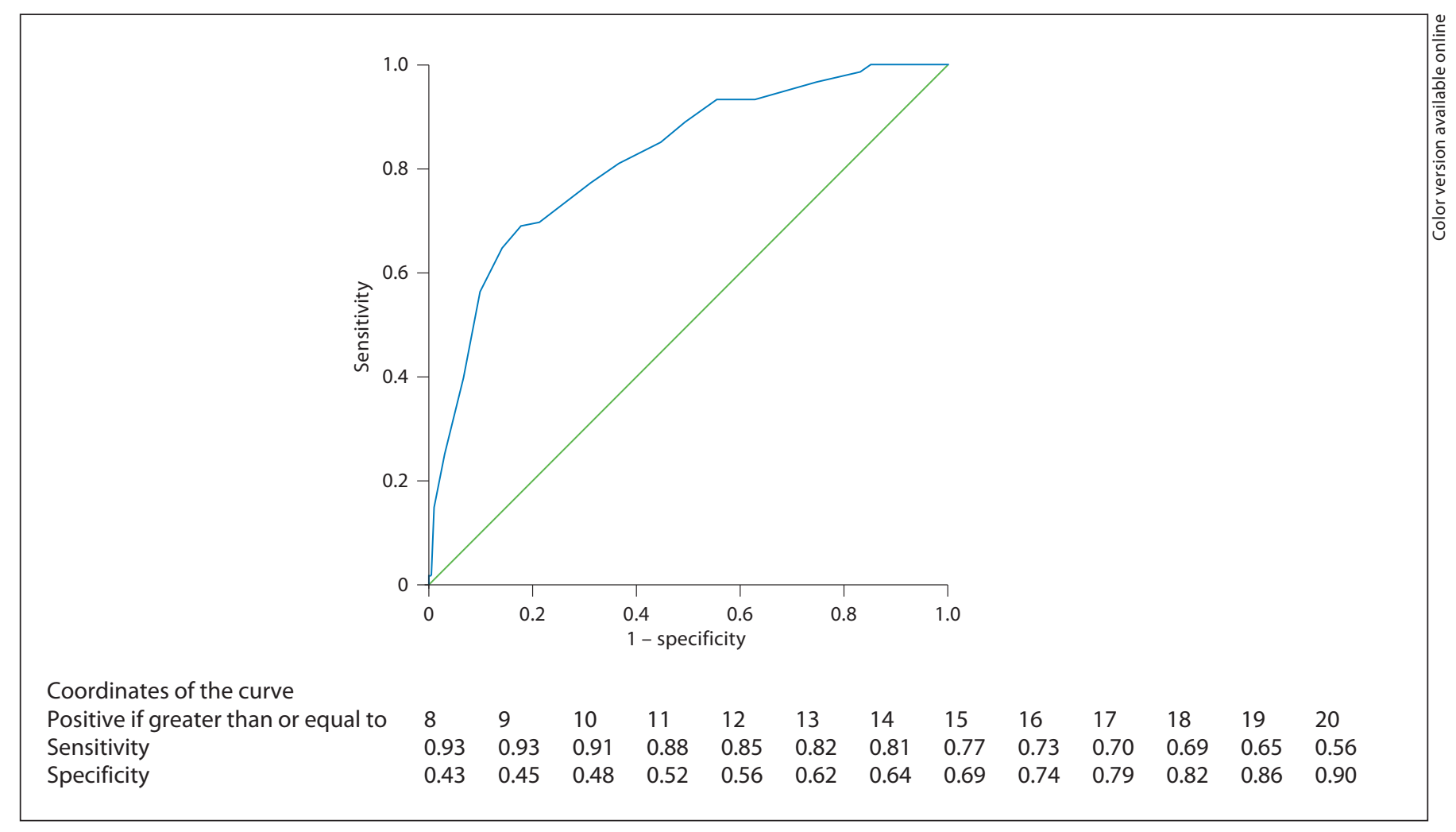

Fig. 1. Receiver operating characteristic analysis of the performance of the HCL-32 in the total sample.

rable with those of samples consisting largely of remitted patients (Italy, Sweden and Spain) [24, 25] and general population samples (Germany and Sweden) [35]. Remarkably, a two-factor structure was also individuated by a large transcultural validation study by Angst et al. [37] in 2010 which involved 12 countries in five geographic regions (total sample $=2,606$ depressed patients; $58 \%$ female), with factor 1 ('active/elated') and factor 2 ('risktaking/irritable') largely unvaried across cultures. Thus, the transcultural validity of the HCL-32 was substantially confirmed, although factor 2 was higher among men and in more severe syndromes, whereas factor 1 was highest in North America and East Europe and lowest in South America. The frequency of the 32 items was substantially stable across different regions except for South Europeans (low symptom frequency in general) and East Europeans (higher reported rates of substance use) [37]. Only the Polish [30] study conducted on 1,051 major depressive patients reported a three-factor structure with the first factor being the biggest and explaining more than half of total variance; it comprised ten items connected with elevated mood and increased activity. The second factor included two items connected with sexual activity, and the third contained three items connected with irritability. However, factor 2 explained $11.8 \%$ of total variance, while factor 3 only $3 \%$. Nevertheless, apart from the separate factor for sexual activity, the results of this study are also in some agreement with those of previous studies. This indicates fairly stable psychometric properties of the HCL-32 regardless of clinical status or cultural differences.

As in preceding studies [35], the first factors would be an indicator of hypomanic symptoms related to increased physical and mental activity, sociability, and self-confidence and the second is related to irritability, distractibility, impulsivity and risk-taking behavior. As expected, increased use of drugs and alcohol are related to this second factor. This dual structure of hypomania with a 'sunny' energetic-euphoric side and a 'dark' irritable-impulsive side was previously described using a preliminary version of the Hypomania Checklist $[9,28]$. We confirm the existence of these two factors and the discriminant analysis indicates that in depressive patients the irritable/distractible/impulsive dimension of the hypomanic 
symptomatology is more important than the hyperactive/elated one for the correct identification of past episodes. This last finding is consistent with the possibility that during depression 'dark' symptomatology might be more easily recognized as pathological in comparison with 'sunny' symptomatology, often interpreted as 'normality' by the patients. Moreover, patients in a depressive episode may be less likely to accurately remember times when they were feeling happy or upbeat (mood-congruent memory bias).

In our sample of patients that completed the HCL-32 during a MDE, the scale exhibits high internal consistency, suggesting that the utility of the scale is not restricted to currently mildly depressed or remitted patients $[24,25]$. Concerning the screening properties, a cutoff point of 14 affirmative responses indicates good sensitivity and specificity for the detection of BD. A HCL32 score of 14 or more was reported by $26.1 \%$ of our cases of DSM-IV-defined MDD. This finding confirms previous observations in clinical samples of patients with mood disorder in different symptomatic phases [24], with even higher rates in the sample screened by Rybakowski et al. [38], where a score of 14 or more was observed in $37.5 \%$ of DSM-IV-defined MDD cases using the Polish version of the HCL-32 [30]. Forty et al. [39] reported that in their sample a HCL-32 cutoff score of 20 offered the best combination of sensitivity (68\%) and specificity (83\%) to discriminate BD-I and MDD recurrent patients, which allowed the detection of bipolarity in $17.2 \%$ of DSM-IV-defined MDD subjects (or up to $32.6 \%$ of DSMIV-MDD cases using a cutoff score of 14). Other authors identified a cutoff of 13 as an optimal balance between sensitivity (0.77) and specificity (0.62) in $\mathrm{BD}$ and unipolar MDE samples [40].

The authors recognized that a cutoff score of 20 was determined by the focus of the study on BD-I patients and by the exclusion of BD-II and MDD recurrent patients with bipolar family history and psychotic features [39]. However, the identification of past hypomanic episodes is particularly important for BD-II and MDD patients reporting depressive symptoms. In these populations, the criterion of 14 affirmative responses to HCL-32 is sensitive enough to alert to the presence of possible BD and therefore may be used in clinical practice for this purpose. Obviously, a more detailed psychiatric assessment is necessary to establish a definite diagnosis.

Interestingly, 175 (39.4\%) of our MDD patients had a HCL-32 score of 14 or above, indicating either some false positives or, alternatively, that hypomanic symptoms may be not considered of diagnostic value in some MDD patients. In this latter group, patients with a HCL-32 profile similar to BD reported more severe depressive symptomatology compared to other MDD patients and more severe anxious symptomatology than BD patients. Regarding hypomanic symptoms, they tend to report a predominant 'dark' irritable-distractible-impulsive profile. It is possible that in these patients irritability, distractibility and impulsivity are not identified as related to a definite period of expansive mood that fulfill the DSMIV diagnostic criteria for (hypo)mania. On the other hand, irritability, distractibility and difficulty in making decisions may often be experienced as part of depression rather than mania, testifying a partial overlap between depressive and manic symptoms. Finally, the presence of a considerably high mean Zung-D total score in our sample, with a substantial rate of patients with a score $>60$ $(\mathrm{n}=232,41.2 \%)$, should also be considered as a potential contributor to a recall bias for past (hypo)manic episodes.

In summary, the HCL-32 has been shown to be a useful instrument for the detection of past hypomania in patients with MDD, although with the possibility of obtaining some false positives. Given the difficulties involved in both the retrospective and cross-sectional diagnosis of hypomania, a key aspect of appropriate management of $\mathrm{BD}$, this questionnaire represents a potential improvement in clinicians' ability to detect and correctly treat $\mathrm{BD}$, especially regarding the use of antidepressant medications in MDD patients actually being bipolar upon administration of the HCL-32 [38].

\section{Come To Me Study Group}

Agrimi Emilia, U.O. Psichiatria 29, 'Istituti Ospitalieri', Cremona; Aguglia Eugenio, U.O. di Clinica Psichiatrica, Università degli Studi di Catania; Amati Amato, Policlinico 'Mater Domini’ - Clinica Psichiatrica Università di Catanzaro; Bogetto Filippo, Azienda Sanitaria Ospedaliera Molinette, Dipartimento di Neuroscienze Università di Torino; Bellomo Antonello, U.O. Psichiatria Ospedale Riuniti di Foggia; Carpiniello Bernardo, Clinica Psichiatrica Università degli Studi di Cagliari; Castrogiovanni Paolo, Policlinico Universitario 'Le Scotte', Clinica Psichiatrica Università di Siena; Colotto Antonio, U.O. Psichiatria USL della Valle di Aosta, Aosta; Costa Raffaele, U.O. Salute Mentale DSM ASL 'Genovese', Genova; Di Fiorino Mario, Reparto di Psichiatria, Ospedale della Versilia, Viareggio (Lucca); Federico Tommaso, AUSL 3 - Dipartimento di Salute Mentale, Catania; Meduri Mario, Clinica Psichiatrica Università degli Studi di Messina; Minnai Gian Paolo, Ospedale S. Martino, Dipartimento di Salute Mentale, Oristano; Nardini Marcello, Dipartimento Scienze Neurologiche Psichiatriche Universitarie, Bari; Placidi Gian Franco, Ospedale Careggi, Dipartimento di Neuropsichiatria Università 
degli Studi di Firenze; Nivoli Giancarlo, Clinica Psichiatrica Università degli Studi di Sassari; Pellegrino Ferdinando, U.O. DSM ASL 'Salerno 1' - Distretto 5C, Salerno; Sartore Luigi, ASL 20 Struttura Complessa Salute Mentale, Alessandria; Sciarretta Antonio, DSM, ASL RMG - Ambulatorio Psichiatrico, Tivoli; Varia Salvatore, DSM modulo $\mathrm{N}^{\circ}$ 7, AUSL Palermo; Viola Marcello, Centro Coordinamento Salute Mentale, Brindisi.

\section{Disclosure Statement}

This study did not involve any drug treatment or devices. The authors report no conflict of interest relevant to this work. Data collection and statistical analysis were supported by Boehringer Ingelheim Italia. Boehringer Ingelheim Italia had no further role in the analysis and interpretation of the data, in the writing of the report or in the decision to submit the manuscript for publication.

\section{References}

$>1$ Bourgeois M: Importance of DSM IV (APA) and ICD-10 (WHO) in diagnosis and treatment of mood disorders (in French). Encephale 1995;21:47-52.

$>2$ Manning JS: Burden of illness in bipolar depression. Prim Care Companion J Clin Psychiatry 2005;7:259-267.

$>3$ Hawton K, Sutton L, Haw C, Sinclair J, Harriss L: Suicide and attempted suicide in bipolar disorder: a systematic review of risk factors. J Clin Psychiatry 2005;66:693-704.

$>4$ Brieger P: Bipolar affective disorders. Part I: diagnosis, epidemiology and course (in German). Fortschr Neurol Psychiatr 2007;75: 673-682, quiz 683-674.

5 Slama F: Epidemiology of bipolar disorders (in French). Rev Prat 2005;55:481-485.

$\checkmark 6$ Berk M, Dodd S, Callaly P, Berk L, Fitzgerald P, de Castella AR, Filia S, Filia K, Tahtalian S, Biffin F, Kelin K, Smith M, Montgomery W, Kulkarni J: History of illness prior to a diagnosis of bipolar disorder or schizoaffective disorder. J Affect Disord 2007;103:181186.

7 Solomon DA, Leon AC, Maser JD, Truman CJ, Coryell W, Endicott J, Teres JJ, Keller MB: Distinguishing bipolar major depression from unipolar major depression with the Screening Assessment of Depression-Polarity (SAD-P). J Clin Psychiatry 2006;67:434442.

$>8$ Benazzi F: Bipolar disorder - focus on bipolar II disorder and mixed depression. Lancet 2007;369:935-945.

$>9$ Akiskal HS, Benazzi F: Atypical depression: a variant of bipolar II or a bridge between unipolar and bipolar II? J Affect Disord 2005;84:209-217.

-10 Akiskal HS, Benazzi F: Continuous distribution of atypical depressive symptoms between major depressive and bipolar II disorders: dose-response relationship with bipolar family history. Psychopathology 2008;41: 39-42.

$>11$ Thase ME: Bipolar depression: diagnostic and treatment considerations. Dev Psychopathol 2006;18:1213-1230.

12 Benazzi F, Akiskal H: Irritable-hostile depression: further validation as a bipolar depressive mixed state. J Affect Disord 2005;84: 197-207.
3 Rybakowski JK, Suwalska A, Lojko D, Rymaszewska J, Kiejna A: Types of depression more frequent in bipolar than in unipolar affective illness: results of the Polish DEP-BI study. Psychopathology 2007;40:153-158.

14 Michalak EE, Murray G, Young AH, Lam RW: Burden of bipolar depression: impact of disorder and medications on quality of life. CNS Drugs 2008;22:389-406.

15 Hirschfeld RM, Williams JB, Spitzer RL, Calabrese JR, Flynn L, Keck PE Jr, Lewis L, McElroy SL, Post RM, Rapport DJ, Russell JM, Sachs GS, Zajecka J: Development and validation of a screening instrument for bipolar spectrum disorder: the Mood Disorder Questionnaire. Am J Psychiatry 2000;157: 1873-1875.

16 Miller CJ, Klugman J, Berv DA, Rosenquist KJ, Ghaemi SN: Sensitivity and specificity of the Mood Disorder Questionnaire for detecting bipolar disorder. J Affect Disord 2004;81:167-171.

17 Cuellar AK, Johnson SL, Winters R: Distinctions between bipolar and unipolar depression. Clin Psychol Rev 2005;25:307-339.

18 Hirschfeld RMA, Holzer C, Calabrese JR, Weissman M, Reed M, Davies M, Frye MA, Keck P, McElroy S, Lewis L: Validity of the Mood Disorder Questionnaire: a general population study. Am J Psychiatry 2003;160: $178-180$.

19 Benazzi F: Improving the Mood Disorder Questionnaire to detect bipolar II disorder (letter to the editor). Can J Psychiatry 2003; 48:770-771.

20 Mago R: Bipolar disorder questionnaire (letter to the editor). Am J Psychiatry 2001;158: 1743.

21 Zimmermann M, Posternak MA, Chelminski I, Solomon DA: Using questionnaires to screen for psychiatric disorders: a comment on a study of screening for bipolar disorder in the community. J Clin Psychiatry 2004;65: 605-610.

22 Hirschfeld RMA, Calabrese JR, Weissman MM, et al.: Screening for bipolar disorder in the community. J Clin Psychiatry 2003;64: 53-59.

23 Angst J, Gamma A, Benazzi F, Ajdacic V, Eich D, Rössler HW: Diagnostic issues in bipolar disorder. Eur Neuropsychopharmacol 2003;13:S43-S50.
24 Angst J, Adolfsson R, Benazzi F, Gamma A, Hantouche E, Meyer TD, Skeppar P, Vieta E, Scott J: The HCL-32: towards a self-assessment tool for hypomanic symptoms in outpatients. J Affect Disord 2005;88:217-233.

25 Vieta E, Sanchez-Moreno J, Bulbena A, Chamorro L, Ramos JL, Artal J, Perez F, Oliveras MA, Valle J, Lahuerta J, Angst J: Cross validation with the Mood Disorder Questionnaire (MDQ) of an instrument for the detection of hypomania in Spanish: The 32 item Hypomania Symptom Check List (HCL-32). J Affect Disord 2007;101:43-55.

26 Benazzi F: Bipolar II disorder and major depressive disorder: continuity or discontinuity? World J Biol Psychiatry 2003;4:166-171.

27 Benazzi F: Is overactivity the core feature of hypomania in bipolar II disorder? Psychopathology 2007;40:54-60.

28 Hantouche EG, Angst J, Akiskal HS: Factor structure of hypomania: Interrrelationships with cyclothymia and the soft bipolar spectrum. J Affect Disord 2003;73:39-47.

29 Bech P, Christensen EM, Vinberg M, BechAndersen G, Kessing LV: From items to syndromes in the Hypomania Checklist (HCL32): psychometric validation and clinical validity analysis. J Affect Disord 2011;132: 48-54.

30 Rybakowski JK, Angst J, Dudek D, Pawlowski T, Lojko D, Siwek M, Kiejna A: Polish version of the Hypomania Checklist (HCL-32) scale: the results in treatment-resistant depression. Eur Arch Psychiatry Clin Neurosci 2010;260:139-144.

31 Perugi G, Canonico PL, Carbonato P, Mencacci C, Muscettola G, Pani L, Torta R, Vampini C, Fornaro M, Parazzini F, Dumitriu A, Come To Me Study Group: Unexplained somatic symptoms during major depression: prevalence and clinical impact in a national sample of Italian psychiatric outpatients. Psychopathology 2011;44:116-124.

32 Zung WW: A self-rating depression scale. Arch Gen Psychiatry 1965;12:63-70.

33 Zung WW: A rating instrument for anxiety disorders. Psychosomatics 1971;12:371-379.

34 Haghighi M, Bajoghli H, Angst J, HolsboerTrachsler E, Brand S: The Farsi version of the Hypomania Check-List 32 (HCL-32): applicability and indication of a four-factorial solution. BMC Psychiatry 2011;11:14. 
35 Meyer TD, Hammelstein P, Nilsson LG, Skeppar P, Adolfsson R, Angst J: The Hypomania Checklist (HCL-32): its factorial structure and association to indices of impairment in German and Swedish nonclinical samples. Compr Psychiatry 2007;48:7987.

36 Wu YS, Angst J, Ou CS, Chen HC, Lu RB: Validation of the Chinese version of the Hypomania Checklist (HCL-32) as an instrument for detecting hypo(mania) in patients with mood disorders. J Affect Disord 2008; 106:133-143.
Angst J, Meyer TD, Adolfsson R, Skeppar P, Carta M, Benazzi F, Lu RB, Wu YH, Yang HC, Yuan CM, Morselli P, Brieger P, Katzmann J, Teixeira Leao IA, Del Porto JA, Hupfeld Moreno D, Moreno RA, Soares OT, Vieta E, Gamma A: Hypomania: a transcultural perspective. World Psychiatry 2010;9: 41-49.

38 Rybakowski JK, Dudek D, Pawlowski T, Lojko D, Siwek M, Kiejna A: Use of the Hypomania Checklist-32 and the Mood Disorder Questionnaire for detecting bipolarity in 1,051 patients with major depressive disorder. Eur Psychiatry 2011, E-pub ahead of print.
39 Forty L, Smith D, Jones L, Jones I, Caesar S, Fraser C, Gordon-Smith K, Craddock N: Identifying hypomanic features in major depressive disorder using the Hypomania Checklist (HCL-32). J Affect Disord 2009; 114:68-73.

40 Yang HC, Yuan CM, Liu TB, Li LJ, Peng HJ, Liao CP, Rong H, Fang YR, Angst J: Validity of the 32-item Hypomania Checklist (HCL32) in a clinical sample with mood disorders in China. BMC Psychiatry 2011;11:84. 